\title{
BOAS PRÁTICAS NA PRODUÇÃO DE MEL NA MICRORREGIÃO DE PAU DOS FERROS.
}

\author{
Pedro Henrique de Assis Silva \\ Discente do curso subseqüente em alimentos \\ Email: henrique_pedro2@hotmail.com \\ Adriana Melo Leite \\ Engenheiro de alimentos \\ Email: adriana.leite@ifrn.edu.br
}

\section{RESUMO}

As Boas Práticas de Fabricação (BPF) e as Boas Práticas Apícolas abrangem um conjunto de medidas que devem ser adotadas pelas unidades de extração do mel a fim de garantir a qualidade sanitária e aconformidade do produto. O presente trabalho teve como objetivo realizar pesquisas na microrregião de Pau dos Ferros, sobre Processo do mel desde a colheita até seu envase. O levantamento foi feito em quatro cidades da região com visitas a apicultores, que foram submetidos a perguntas relacionadas ao modo de colheita do mel e seu processamento. As pesquisas mostram que, a apicultura na região esta evoluindo, mas precisa de melhorias.

PALAVRAS CHAVES: Mel, BPF, BPA, pesquisa.

\section{GOOD PRACTICES IN THE HONEY PRODUCTION IN THE MICRO REGION OF PAU DOS FERROS}

\begin{abstract}
The Good Practices of Production (GPP) and the Good Practices of Apiculture involve a group of measures that must be adopted by units of honey extraction in order to guarantee the sanitary quality and the conformity of the product. The present work aimed to do researches in the micro region of Pau dos Ferros about the processing of honey since its harvest until its packaging. The rising was done in four cities of the region with visits to beekeepers, who were submitted to questions related to the way of honey harvest and its processing. The researches show that the apiculture in the region is developing, but it needs improvements.
\end{abstract}

KEY-WORDS: Honey, GPP, GPA, research 


\section{BOAS PRÁTICAS NA PRODUÇÃO DE MEL NA MICRORREGIÃO DE PAU DOS FERROS.}

\section{INTRODUÇÃO}

A microrregião de Pau dos Ferros é uma das microrregiões do estado brasileiro do Rio Grande do Norte pertencente à mesorregião Oeste Potiguar. Sua população foi estimada em 2006 pelo IBGE em 116.160 habitantes e está dividida em dezessete municípios. Possui uma área total de $2.672,604 \mathrm{~km}^{2}$.

A criação de abelhas é hoje uma importante atividade agropecuária no Brasil, representando trabalho e renda para muitas famílias de pequenos e médios produtores rurais. Dos produtos obtidos da colméia, o mel é o mais importante, sendo o principal objetivo da exploração apícola brasileira, composto basicamente de carboidratos, é considerado um alimento de alto valor energético para o organismo humano, possui ainda vários minerais, proteínas, ácidos orgânicos, vitaminas, hormônios, enzimas e pigmentos vegetais. (CRANE, 1987).

A participação do setor apícola brasileiro no mercado internacional provocou mudanças em toda a cadeia produtiva da apicultura, sendo a busca por qualidade uma das mais observadas. Isso se deve à necessidade do atendimento de normas que regulamentam o comércio de alimentos entre os países do mundo. Essas normas são estabelecidas em fórum internacional e têm o propósito de garantir a comercialização de alimentos seguros. A aplicação destas é uma garantia à saúde dos consumidores dos países importadores, pois sabem que, ao usar os produtos, não estarão pondo em risco sua saúde. Diante das exigências do mercado internacional, a qualidade do mel produzido no Brasil melhorou muito, mas algumas melhoras ainda são necessárias. Atualmente não basta provar, através de análises em laboratórios, que o produto é de boa qualidade. Temos que comprovar e garantir que nosso produto é seguro e que pode ser consumido sem risco à saúde. Para tanto, são necessários que sejam estabelecidos, descritos e registrados os procedimentos realizados desde a produção até o consumo, com o objetivo de assegurar a qualidade do mel que vai do campo à mesa do consumidor

(SEBRAE, 2009).

Este trabalho foi desenvolvido para fazer um levantamento do uso das Boas Práticas na Apicultura do campo até a unidade de processamento na obtenção de um mel de qualidade. Visto que a região Nordeste possui uma das melhores floras apícolas do mundo, e o estado potiguar que exportou em $20091.951 .000 \mathrm{Kg}$, aparece como $6^{\circ}$ maior exportador brasileiro segundo o SEBRE/UAGRO.

Para realização dessa pesquisa foram visitados quatro municípios: Pau dos Ferros, José da Penha, Portalegre, Rafael Fernandes.

\section{MATERIAL E MÉTODOS}

Foram utilizadas duas listas de verificação para realização da pesquisa, uma baseada no Manual de Boas Práticas Apícolas (SEBRAE, 2009) e outra na legislação de BPF (Portaria SVS/MS no 326/97). A lista de verificação elaborada para o campo discrimina o local de instalação do apiário, utensílios, indumentária apícola, uso da fumaça e transporte até a unidade de processamento (Casa do Mel) enquanto o chek list para a Casa do Mel discrimina sobre as áreas externa e interna; o acesso a casa do mel; estado de conservação dos tetos; 
paredes, portas e janelas; instalações sanitárias para os manipuladores; lavatórios na área de produção; iluminação e instalação elétrica; ventilação e climatização; higienização das instalações, utensílios e equipamentos; controle integrado de vetores e pragas urbanas; abastecimento de água; manejo dos resíduos; esgotamento sanitário; leiaute; avaliação dos manipuladores (vestuários, hábitos higiênicos, estado de saúde, capacitação e supervisão); e ainda avaliação do produto (transporte, fluxo de produção, rotulagem e armazenamento, controle de qualidade do produto final).

\section{RESULTADOS E DISCUSSÃO \\ BOAS PRÁTICAS APÍCOLAS - CAMPO}

\section{Apiário}

Após as pesquisas com apicultores da região verificou-se que os apiários da região possuem entre 10 e 30 colméias, com distância de $2 \mathrm{~m}$ de uma para outra como exige a legislação vigente, não utilizam alimentação artificial, estão em área sombreada a mais de $300 \mathrm{~m}$ de residências, currais, galpões de criação e estradas, possuem distância de mais de $3.000 \mathrm{~m}$ de lixões, aterros sanitários etc, se encontram próximo a fontes naturais de néctar e pólen. Em um dos apiários visitados, os próprios apicultores fabricavam as colméias, não atendo as recomendações exigidas.

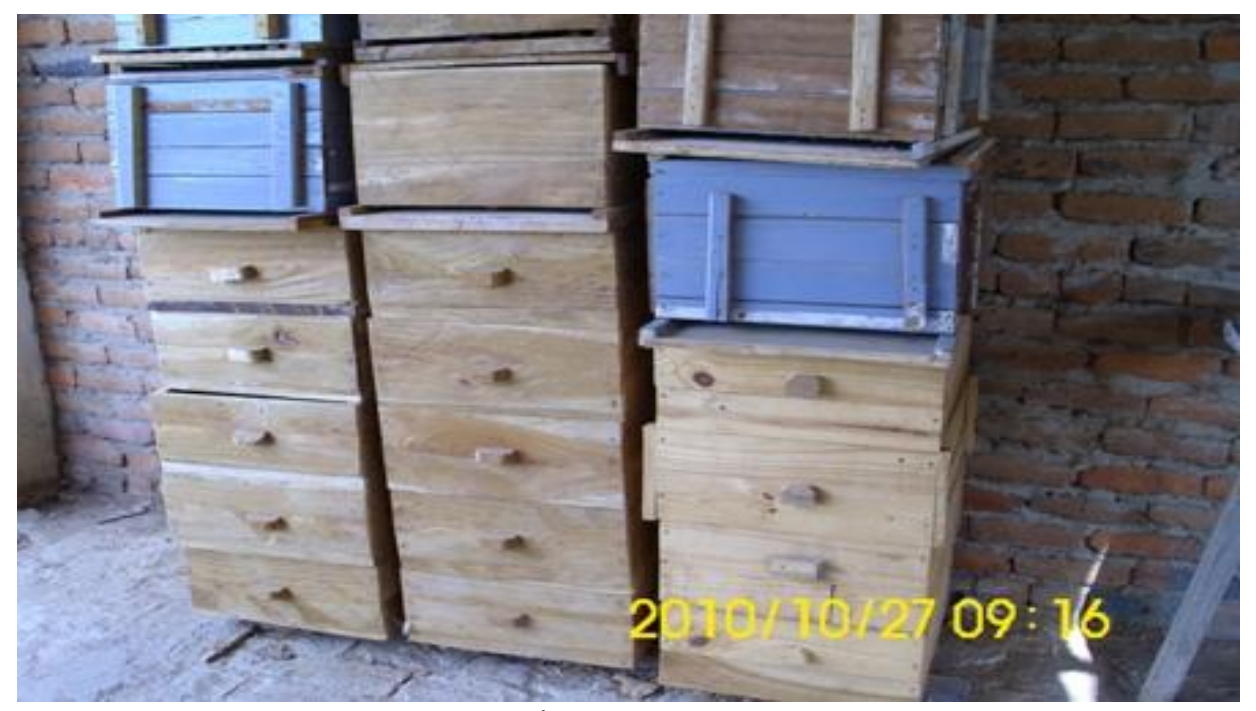

Figura 1 - Colméias sem espaço nas laterais

Para fabricar uma boa colméia é necessário que ela assegure boas condições para as abelhas, e uma boa segurança para o mel, evitando assim alterações.

\section{Manejo}

A garantia da produção de um alimento seguro deve ser o objetivo de todos que atuam na cadeia produtiva do mel e compromisso assumido pelos apicultores, que são os responsáveis pela produção da matéria prima dos entrepostos de mel. Com a aplicação das Boas Práticas Apícolas se tem a redução dos riscos de contaminação e o controle dos perigos que comprometem a qualidade e inocuidade do mel. Esse cuidado proporciona segurança à saúde do consumidor e amplia as possibilidades de comercialização do mel produzido, conferindolhe maior competitividade. A produção de mel seguro e de qualidade é uma exigência de 
mercado e um diferencial decisivo para assegurar competitividade ao setor apícola brasileiro, devendo, por isso ser rigorosamente aplicado no campo (SEBRAE, 2009).

No manejo, foram encontrados os principais riscos para a não obtenção de um mel de qualidade, alguns apicultores colhem durante a noite podendo causar alteração no teor de umidade, não utilizam formão em inox, sendo utilizado até mesmo facão, alguns colocam as colméias diretamente no solo podendo haver risco de contaminação, o transporte das melgueiras é realizado em carro de mão, moto e carro aberto cobertos com lona, não sendo de uso exclusivo para apicultura.

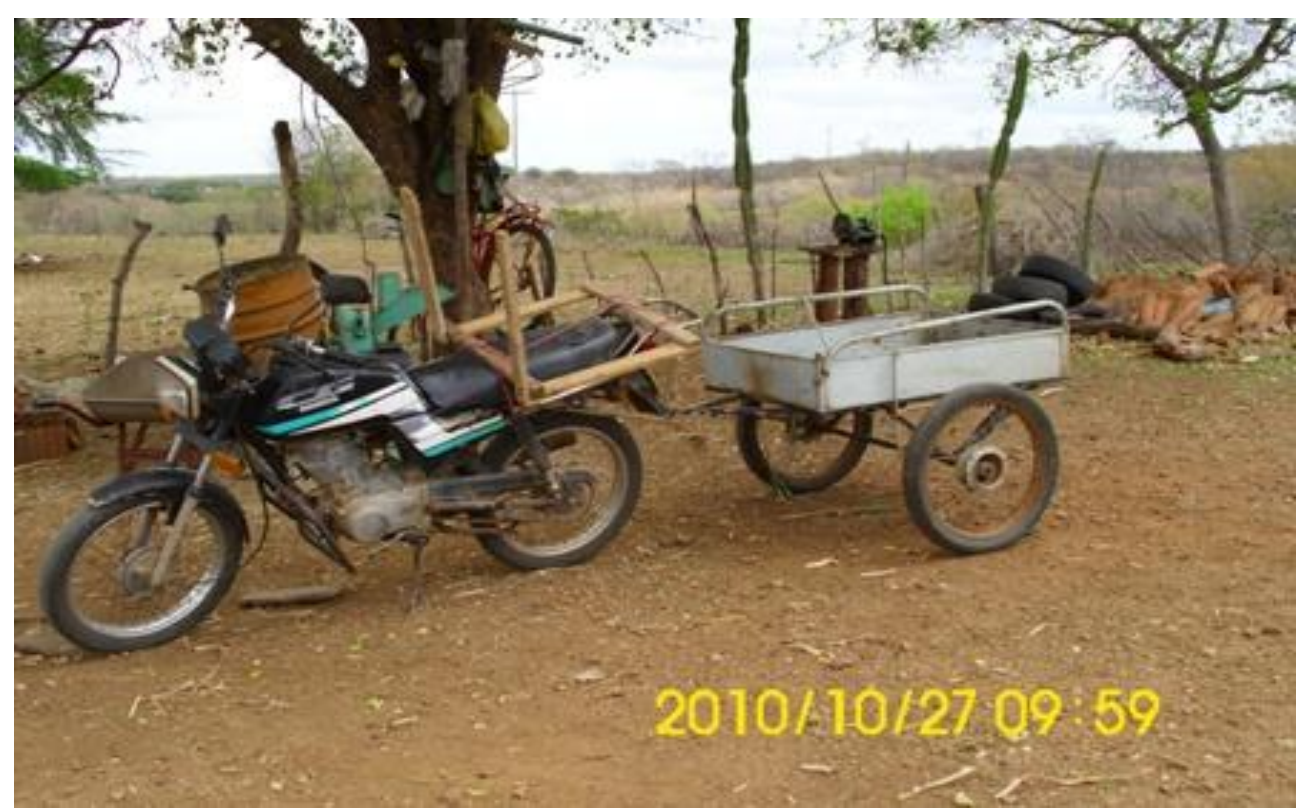

Figura 4 - Moto utilizada no transporte de melgueiras

Destaca-se também pontos positivos, a maioria utiliza a fumaça corretamente em quantidade e qualidade, todos colhem os favos sem presença de crias e totalmente operculados, garantindo um mel maduro com teor de umidade adequado, a fumaça utilizada é fria e limpa, não sendo direcionada diretamente nos favos conservando as características sensórias do mel, os favos são mantidos tampados, o apicultor utiliza indumentária adequada (macacão, máscara, luva e bota), as pessoas que trabalham com a colheita das colméias no campo não entram na área de manipulação na casa do mel e as melgueiras também não entram na área de processamento.

\section{VERIFICAÇÃO DAS BOAS PRÁTICAS DE FABRICAÇÃO EM ESTABELECIMENTOS PRODUTORES/ INDUSTRIALIZADORES DE ALIMENTOS (BPF).}

Foram encontradas poucas casas de mel em funcionamento na região, muitos apicultores retiram o mel em locais improvisados sem as condições higiênicas necessárias. Um dos grandes problemas, segundo os apicultores, para a construção de unidades de processamentos, está na planta que é entregue a eles com deficiências, visto que sua construção deve atender a exigências legais referente às condições higiênico-sanitárias determinadas em lei, pelo Ministério de Agricultura Pecuária e Abastecimento (MAPA), para estabelecimentos elaboradores e industrializadores de alimentos (Portaria SIPA ${ }^{\circ} 006$, de 25 de julho de 1985 e Portaria n n $^{368}$, de 04 de setembro de 1997 - DIPOA). 
De acordo com a lista de verificação das boas praticas em estabelecimentos verificou-se nas casas de mel existentes em funcionamento as seguintes características: possuem área externa e interna com objetos em desuso, muitos com poeira e com focos de insalubridade, as vias de acesso não possuem superfície pavimentada, os pisos são resistentes ao impacto, impermeável, laváveis e de fácil limpeza, paredes e tetos estão nas medidas recomendáveis, portas e janelas são de madeira, facilitando a proliferação de vetores e pragas como cupins, enquanto deveriam ser metálicas ou revestidas com material Impermeável:

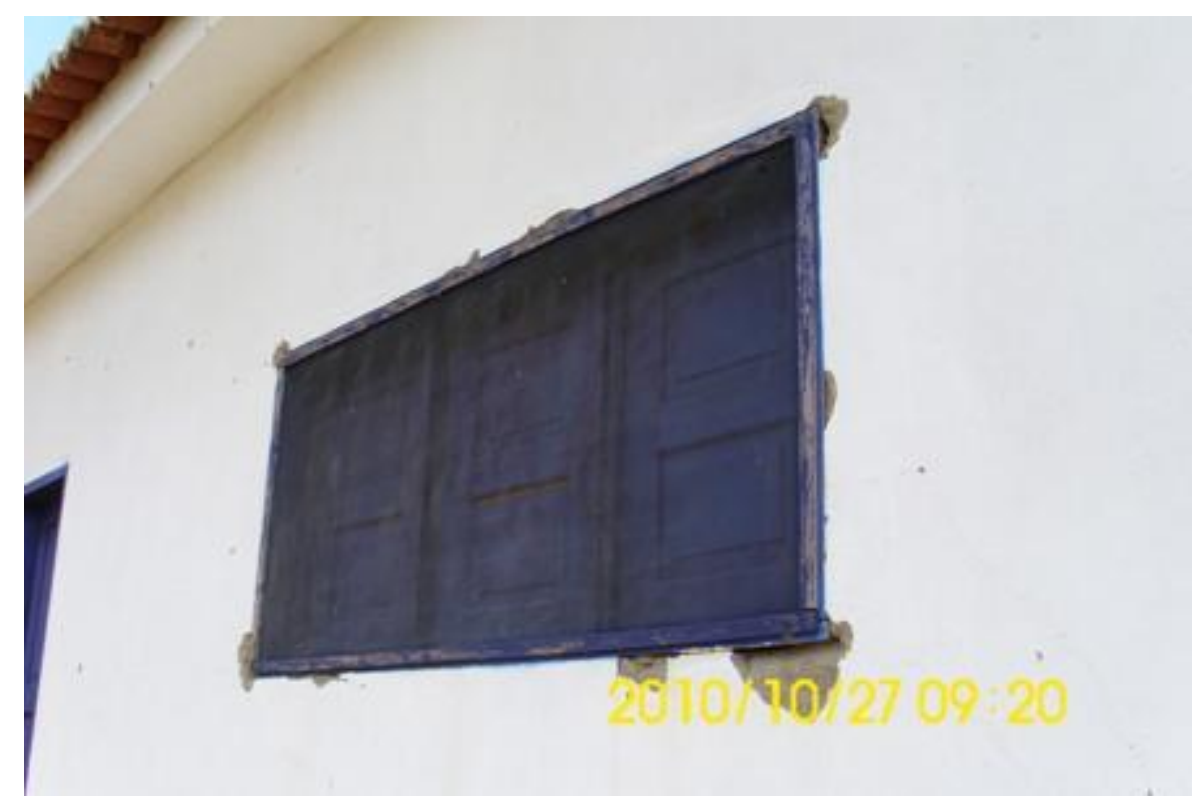

Figura 5 - Janela de madeira telada

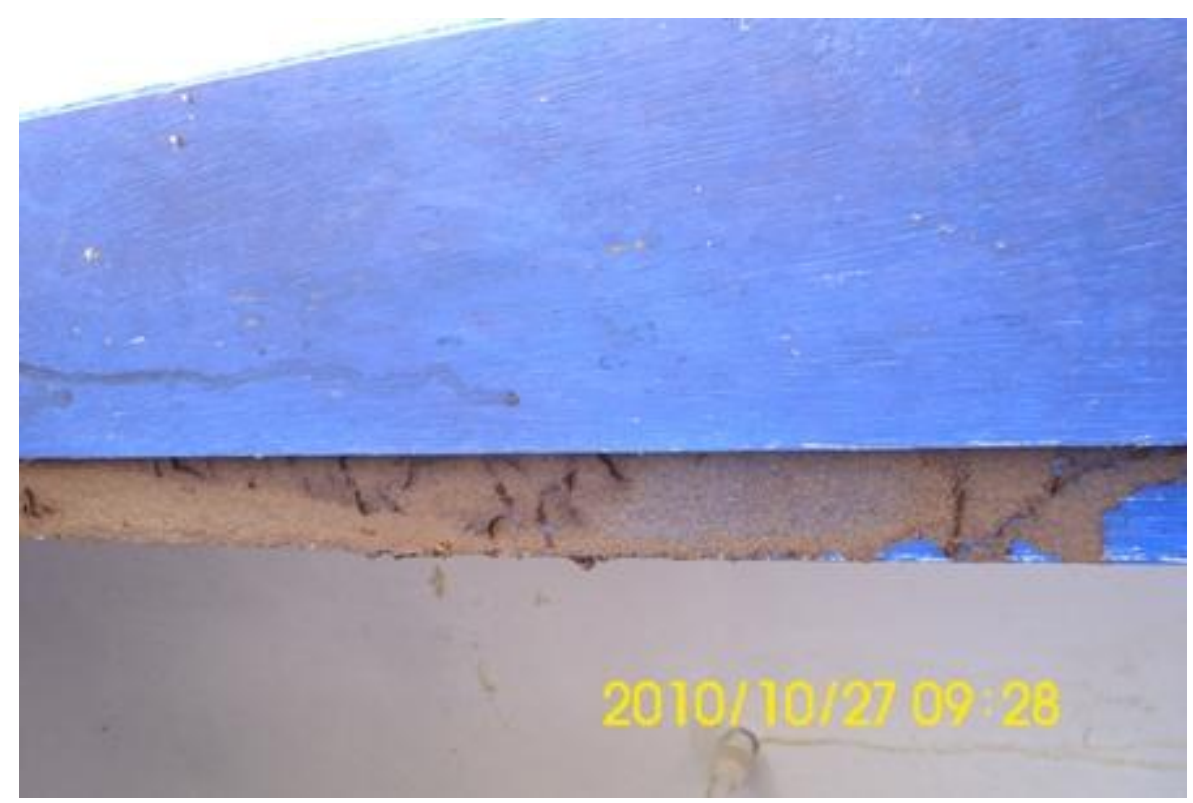

Figura 6 - Proliferação de cupins nas janelas

A água é de boa qualidade, os banheiros e vestuários onde os manipuladores higienizam-se lavando as botas e mãos, não possuem comunicação direta com as áreas de manipulação e recepção do mel, a iluminação é adequada, suprindo as necessidades sem comprometer a qualidade do produto, a ventilação é natural. 


\section{Equipamentos e utensílios}

A maioria dos equipamentos e utensílios empregados são inox como é recomendado, não permitindo a liberação de substâncias toxicas, odores nem sabores no produto, as superfícies são lisas e isentas de imperfeições. A seguir imagens dos equipamentos utilizados:

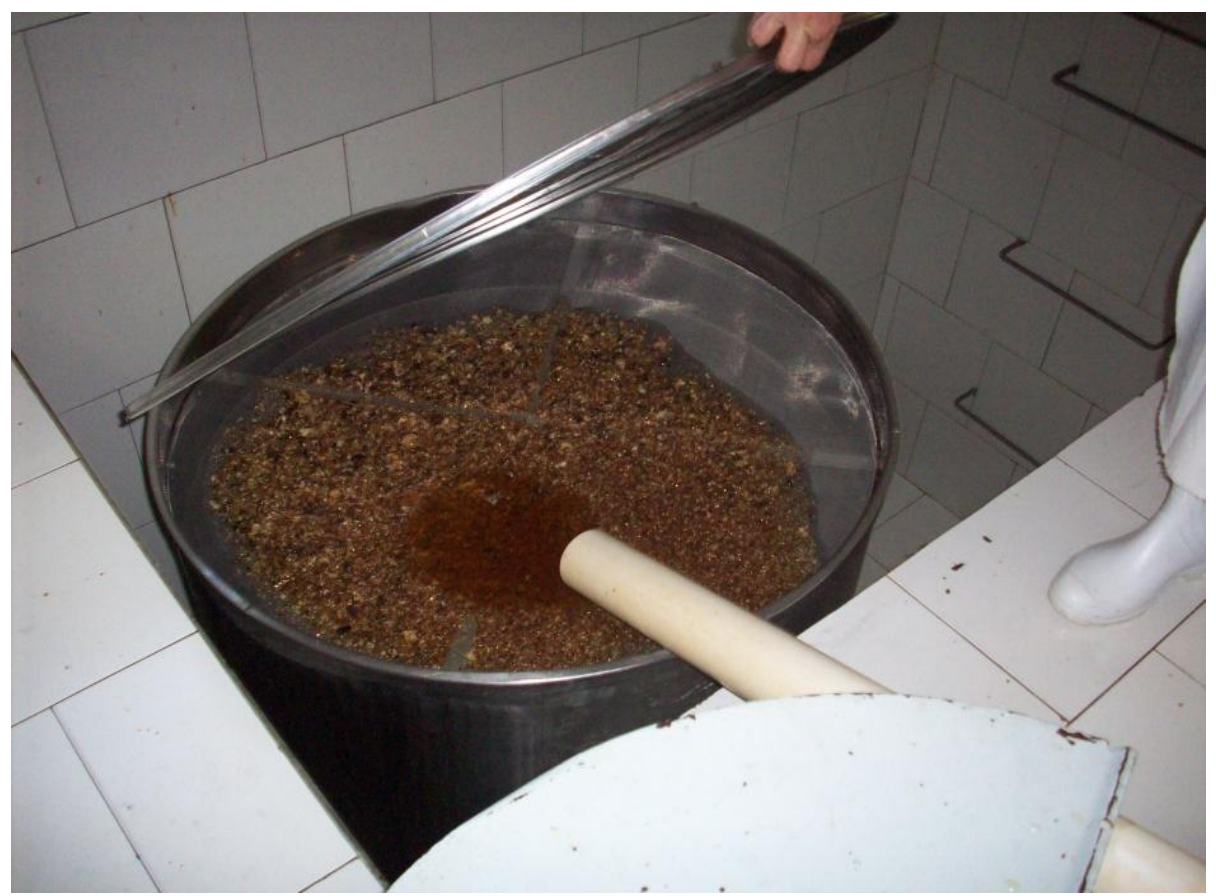

Figura 7 - Decantador

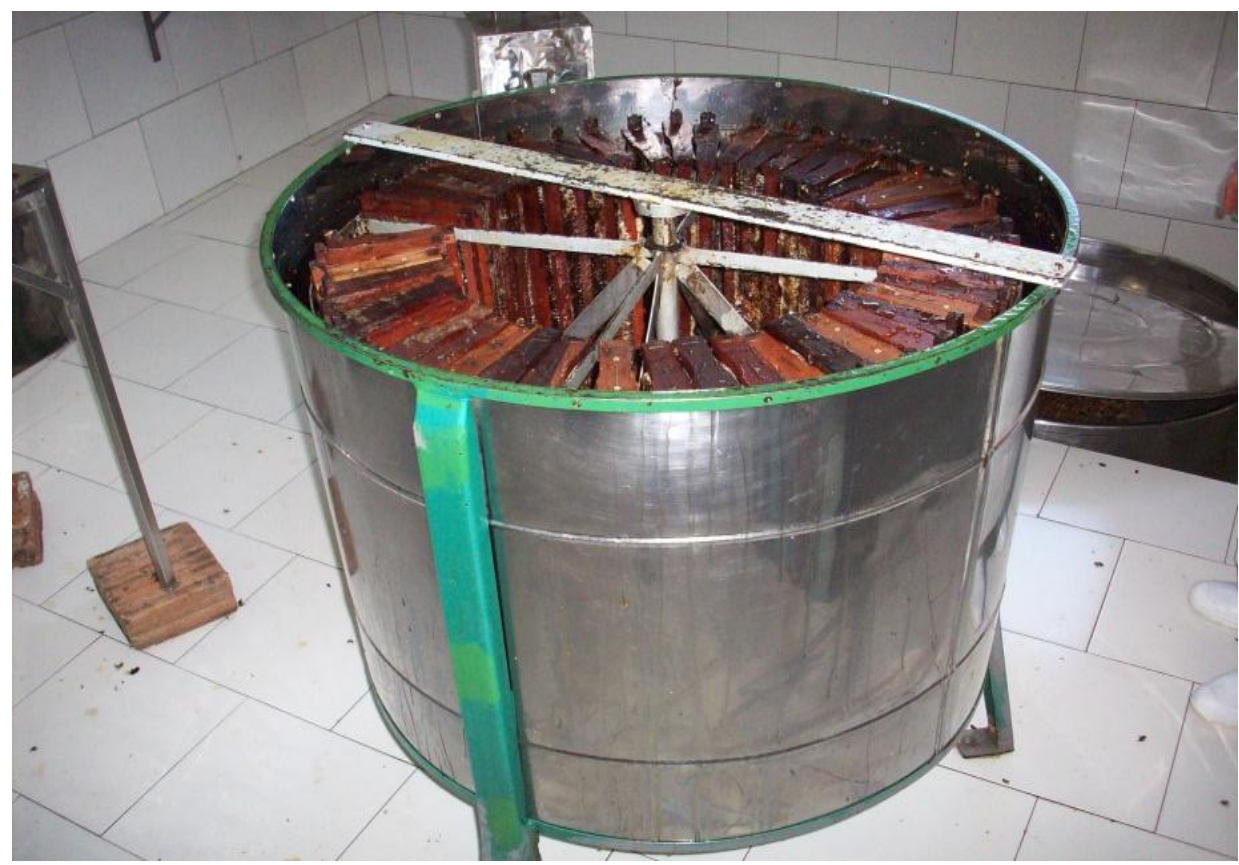

Figura 8 - Centrifuga mecânica 


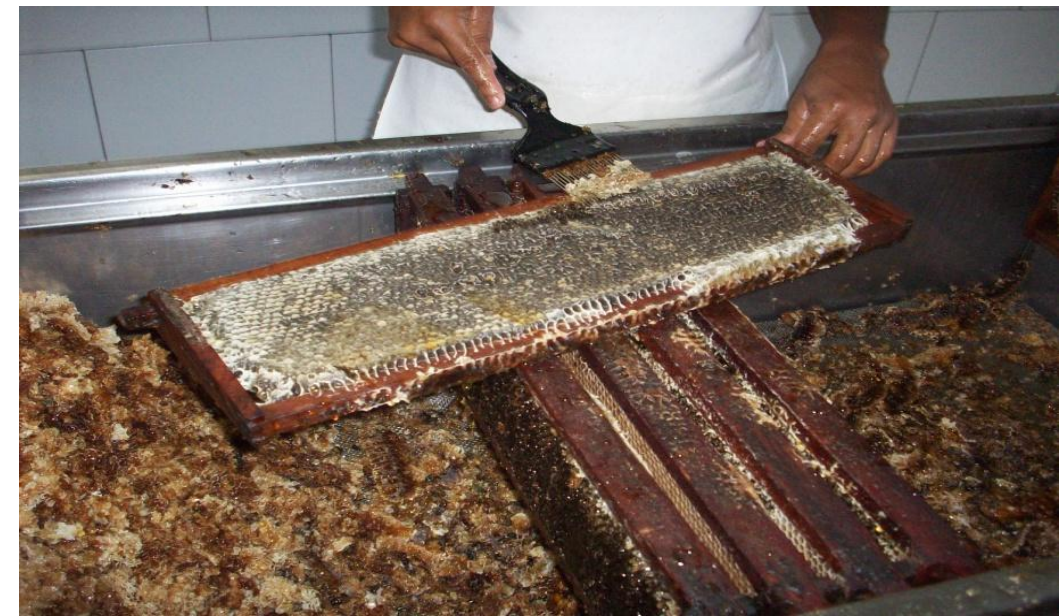

Figura 9 - Desoperculação

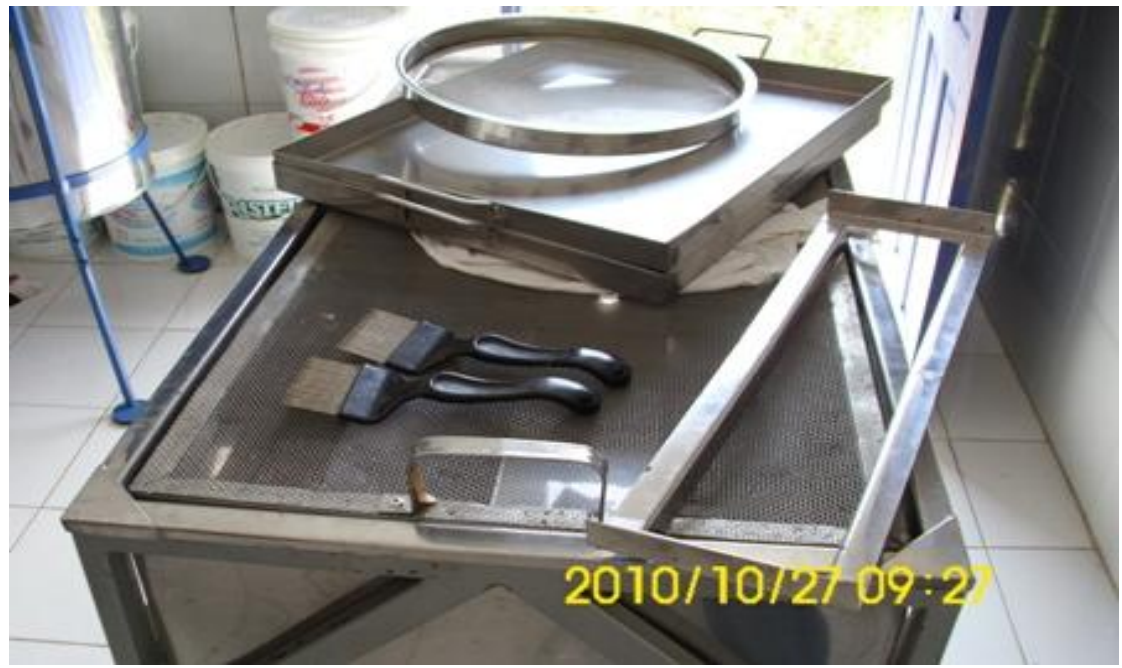

Figura 7 - Equipamentos apícolas

Apenas em uma das casas de mel foi encontrada uma centrifuga com partes de ferro, podendo comprometer a qualidade do mel.

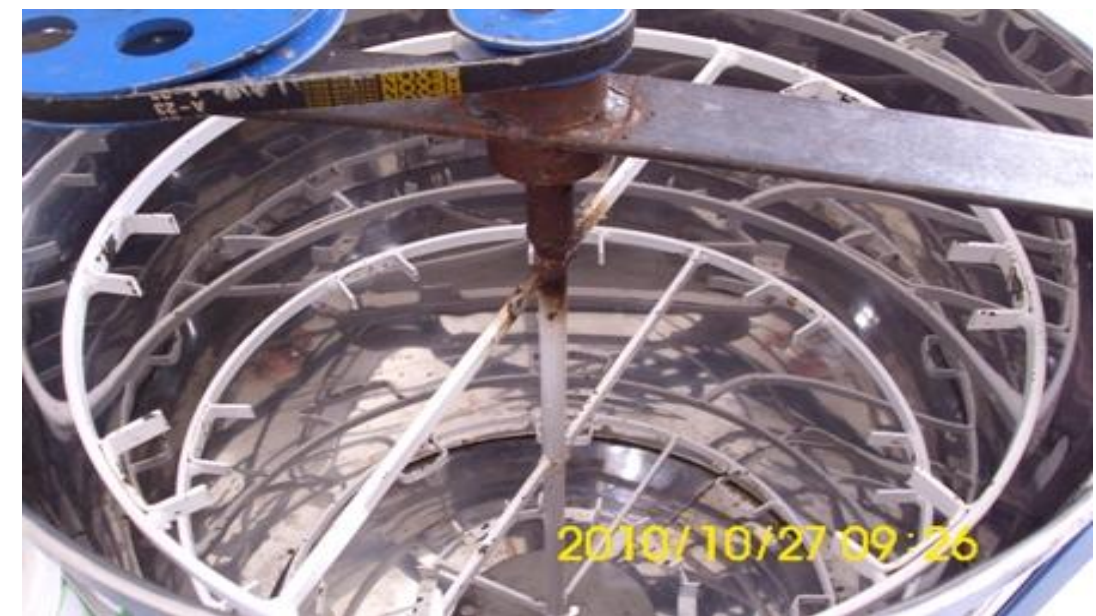

Figura 8 - Centrifuga Manual 


\section{CONCLUSÃO}

Verificou-se nessas visitas que a atividade apícola na região ainda tem muito a melhorar, as pessoas não se sentem motivadas pela apicultura, e não investem na qualidade do produto, talvez por falta de divulgação de quanto o mel é importante, tanto socialmente, quanto economicamente.

A próxima etapa será continuar o levantamento em outras cidades para ter uma visão maior da apicultura na microrregião de Pau dos Ferros, e em seguida trabalhar com os apicultores o uso das Boas Práticas na produção de mel através de capacitações e palestras.

\section{REFERÊNCIAS}

1. CRANE,E. O livro do mel. 2 ed. São Paulo. Nobel, 1987, 226 p

2. SEBRAE Nacional (Brasília, DF)

3. ROGÉRIO A. Pereira, Luciene Xavier de Mesquita, Lionel Segui Gonçalves, David de Jong e Katia P.Gramacho' Mensagem Doce $n^{\circ} 106$ Maio de 2010, disponível em:< file:///E:/PROJETO\%20MEL/artigo\%20mel\%20referencia.htm

4. Regulamento técnico sobre as condições higiênico-sanitárias de Boas Práticas de Fabricação para estabelecimentos elaboradores/industrializadores de alimentos Portaria DIPOA no 368, de 04 de setembro de 1997. Brasília: MA/DAS/DIPOA/DNT, 1997.

5. ANVISA, 2003: disponível em: $<$ http://www.anvisa.gov.br/alimentos/bpf.htm

6. Magalhães, E. de Oliveira - MSc Ceplac/Cepec/Secen/Apicultura 2003

7. IBGE, 2006: disponível em:<http://pt.wikipedia.org/wiki/Microrregi\%C3\%A3o_de_Pau_dos_Ferros

8. Sousa, Darcet Costa - Org: Apicultura: Manual do agente de desenvolvimento rural/ Organizado por Darcet Costa Sousa._2.ed.rev.._Brasília: SEBRAE, 2007 